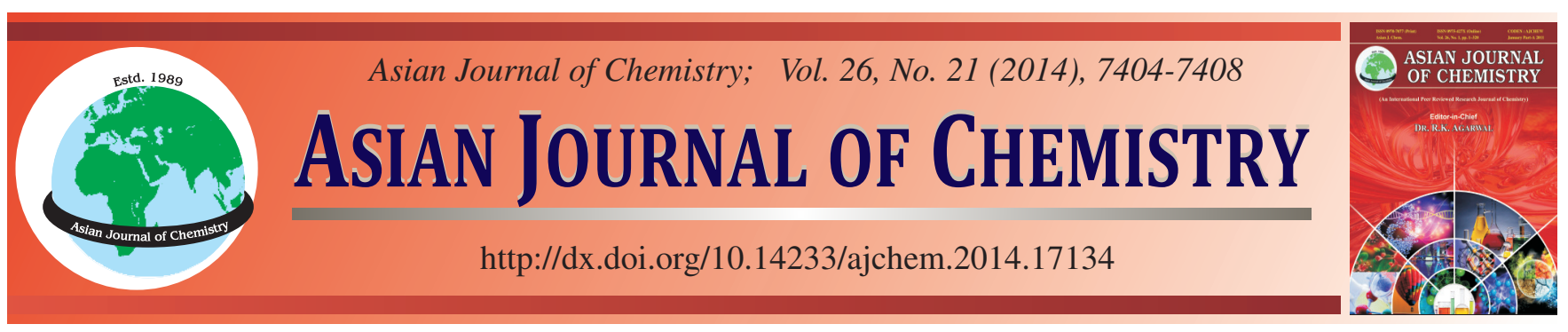

\title{
Computational Study on the Second-Order Nonlinear Optical Properties of Coumarin Derivatives with N-p-Vinylphenyl Carbazole Chromophores
}

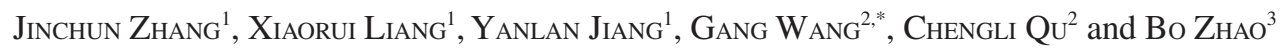

\begin{abstract}
${ }^{1}$ Department of Basic Sciences, Naval Aeronautical and Astronautical University, Yantai 264001, Shandong Province, P.R. China ${ }^{2}$ Yantai Institute of Coastal Zone Research, Chinese Academy of Sciences, Yantai 264003, Shandong Province, P.R. China ${ }^{3}$ School of Chemistry and Environmental Science, Nanjing Normal University, Nanjing 210097, Jiangsu Province, P.R. China

*Corresponding author: Fax: +86 535 2109000; Tel: +86 535 2109019; E-mail: gwang@yic.ac.cn
\end{abstract}

\begin{abstract}
Based on the density functional theory, a computational study was performed to investigate the second-order nonlinear optical properties of ten coumarin derivatives with $\mathrm{N}$ - $p$-vinylphenyl carbazole chromophores. All tested derivatives were divided into two groups, one with different substituent groups on carbazole (named as compounds b1 to b4) and the other with the N-p-vinylphenyl carbazole substituent modified at different positions on coumarin (named as compounds a to $\mathbf{f}$ ). The geometrical structure of these derivatives were first comprehensively optimized through density functional theory method at B3LYP/6-311G level. Then their static second-order nonlinear optical polarizabilities $(\beta)$ were calculated at the same level and the molecular electric spectrum of each derivative was obtained via the time dependent density functional theory (TD-DFT). Computational results show that all these derivatives have large $\beta_{\text {tot }}$ values and excellent transparence. However, as compared to compounds a-f, compounds b1-b4 have longer conjugated bridges and larger $\beta_{\text {tot }}$ values and show better planarity. It suggests that low transition energy, large $\Delta \mu_{\mathrm{eg}}$ values and large charge transfer range play vital roles in the high second-order nonlinear optical response.
\end{abstract}

Keywords: Coumarin, Carbazole, Density functional theory, Second-order nonlinear optical property.

\section{INTRODUCTION}

With the development of laser technology, nonlinear optical materials, as key materials for photonic communication and digital memory, have been extensively applied to industry, national defense, medicine, research, etc. ${ }^{1,2}$. Among them, organic nonlinear optical materials draw much more attentions than those inorganic and arouse extensive interests. Such organic materials were characterized for high first hyperpolarizability values $(\beta)$, shorter response time, lower dielectric constants, and high photoelectric coefficients. On the other hand, they are more flexible due to their diverse modifiability and variable synthetic strategies ${ }^{3-5}$.

Theoretically, if high nonlinear optical responses were achieved, a second-order nonlinear optical molecule should have a large $\beta$ value. However, for most organic molecules, their nonlinear optical properties are interdependently related with their spectral transmission properties. Molecules with larger $\beta$ values generally possess longer maximum absorption wavelengths $\left(\lambda_{\max }\right)$ and shorter transparence ranges. The design of molecular materials with large $\beta$ values, blue-shift absor- ption and high thermo-stability has widely concerned ${ }^{6-8}$. It was reported that the introduction of heterocycles would significantly enhance nonlinear optical responses but slightly affect the transparence. The mechanism was attributed to the orientation relation between auxiliary donors (electron-excessive heterocyclic bridges) and auxiliary acceptors (electrondeficient heterocyclic bridges), which alternatively lead to the increase of $\beta$ values.

Benefited from their unique optical characteristics, coumarins have been developed into an important type of nonlinear optical materials. Such organic compounds were characterized by high emissivity, light colour, excellent light transmittance, extended spectral range, high thermo-stability, $e t c^{9-11}$. In this study, a particular emphasis was devoted to coumarin derivatives modified with $\mathrm{N}$ - $p$-vinylphenyl carbazole chromophores. Such chromophores have conjugated rigid plane structures and electron-excessive heterocyclic rings, which could potentially promote the second-order nonlinear optical properties. The objective of this paper was to investigate the likely promotion via computations based on DFT and TDDFT method. 


\section{COMPUTATIONAL DETAILS}

Design of molecules: Two types of coumarin derivatives with $\mathrm{N}$ - $p$-vinylphenyl carbazole chromophores (Fig. 1), one with $\mathrm{N}$ - $p$-vinylphenyl carbazole substituent modified at different positions on coumarin (named as compounds a to $\mathbf{f}$ ) and the other with different substituent groups on carbazole (named as b1 to b4), were designed in this study. Compounds a to $\mathbf{f}$ were designed to investigate the influences of substituent positions on electron migration and consequential changes in second-order nonlinear optical responses. Compounds b1 to b4 were designed to investigate the influences of different substituent groups on the conjugated structure and second-order nonlinear optical properties.

Design of computation: The DFT has become a popular method in calculating molecular properties such as molecular energies, ground state geometries, and optical transitions due to the high accuracy. The best DFT method achieves significantly greater accuracy than Hartree-Fock theory at only a modest increase in cost (far less than MP2 for medium size and larger molecular systems $)^{12-14}$. It does so by including some of the effects of electron correlation much less expensively than traditional correlated methods. The geometries of all designed molecules were optimized by DFT methods at the B3LYP/6-311G level to minimize E. Corresponding parameters like the dipole moment $(\mu)$, and the static first hyperpolarizability $\left(\beta_{\text {tot }}\right)$ were calculated by the same method.

On the other hand, to obtain the relationship between the electronic structure and second-order nonlinear optical properties of the molecules, the electronic absorption parameters, including wavelengths, oscillator strengths, and main assignment, were systematically investigated by using TD-DFT method on the basis of the optimized ground structures. TDDFT is one of the most popular methods for the calculation of excitation energies in quantum chemistry due to its efficiency and accuracy. The calculation of the natural bond orbital (NBO) analysis is performed at the B3LYP/6-311G level. All of the calculations in this work were carried out by using Gaussian 03 program package.

\section{RESULTS AND DISCUSSION}

Geometrical structures and charges distributions: Fig. 1 demonstrates the optimized geometrical structures of ten designed molecules in cartesian coordinate system. It is noteworthy that all these structures are nonplanar. The dihedral angel between the carbazole fragment and the styryl fragment, i.e. $\angle \mathrm{C} 10-\mathrm{N} 9-\mathrm{C} 12-\mathrm{C} 13$, ranges from $64^{\circ}$ to $68^{\circ}$ for compounds a to $f$ while $49^{\circ}$ to $58^{\circ}$ for compounds b1 to b4. On the other hand, the large dihedral angels between the styryl fragment and coumarin, i.e. $\angle \mathrm{C} 14-\mathrm{C} 15-\mathrm{C} 16-\mathrm{C} 17$ were only observed for compounds $\mathbf{d}$ and $\mathbf{e}\left(30^{\circ}\right.$ and $38^{\circ}$, respectively) while other angels were restricted within $4^{\circ}$. As a whole, the structural coplanarity of compounds b1 to b4 is relatively better than that of compounds a to f, which suggests the better conjugacy for compounds b1 to b4. Among all designed molecules, compounds $\mathrm{d}$ and e are considerably poor in both coplanarity and conjugacy. It suggests that either the forms or the positions of substituents would lead to remarkable variation in conjugacy.

Atom charge is different from electron density, so it could not be observed by using quantum mechanics. As an absolute ordain, its results can reflect the static state atom charge distribution in the molecule to a certain extent. For a noncentrosymmetric molecule, the second-order polarizability caused by charge transfer $\left(\beta_{\mathrm{CT}}\right)$ of the whole system would generally get enhanced by the strong intramolecular charge transfer (CT). As a result, better nonlinear optical activities could be further achieved. In this paper, each designed molecule was artificially divided into three parts to evaluate the charge distribution, i.e. Part I for substituents (carbazole together with its substituents), Part II for styryl, and Part III for coumarin. The ground state charge distributions of all compounds, which obtained via natural bond orbital methods, were presented in Table-1. It is

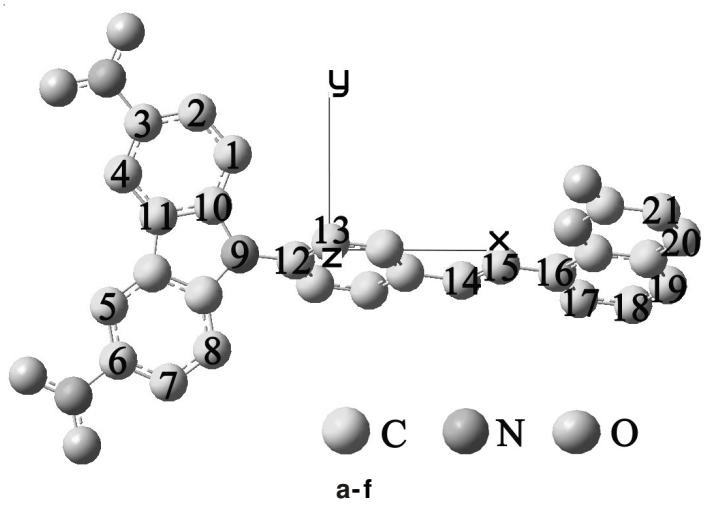

The positions of 3,6-dinitro-9-(4-vinylphenyl)-carbazole on coumarin: a: 16; b: 17; c: 18; d: 19; e: $20 ;$ f: 21

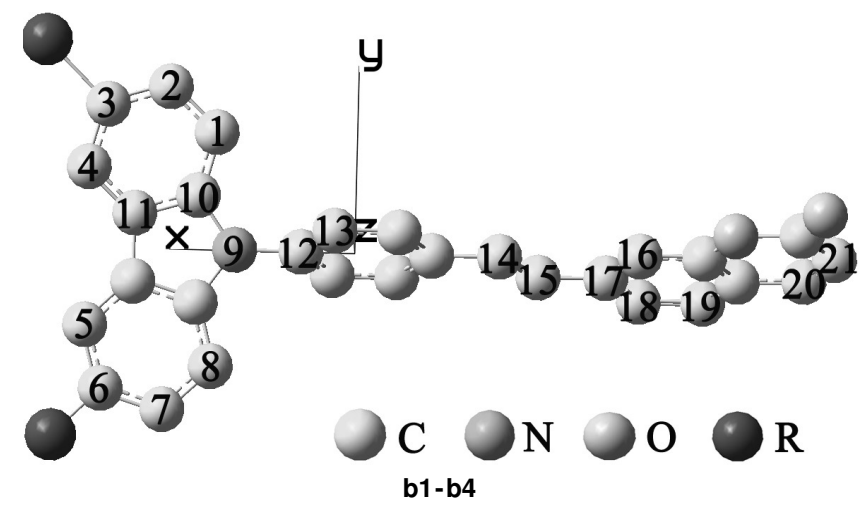

$\mathrm{b} 1: \mathrm{R}=\mathrm{NH}_{2} ; \mathrm{b}_{2}: \mathrm{R}=\mathrm{OH}$; b3: $\mathrm{R}=\mathrm{OCH}_{3} ; \mathrm{b}_{4}$ : R=br

Fig. 1. Optimized geometry structures of the studied molecules at the B3LYP/6-311G level of theory showing the atom labeling scheme and Cartesian axes

\begin{tabular}{|c|c|c|c|c|c|c|c|c|c|c|}
\hline & NATUI & L BOND & RBITAI & BO) $\begin{array}{r}\mathrm{TA} \\
\mathrm{CH}\end{array}$ & GE DIST & 3UTION & $\mathrm{FF}$ EACl & RT & & \\
\hline Molecule & $\mathrm{a}$ & $\mathrm{b}$ & $\mathrm{c}$ & $\mathrm{d}$ & $\mathrm{e}$ & $\mathrm{f}$ & b1 & b2 & b3 & $\mathrm{b} 4$ \\
\hline I & -0.259 & -0.247 & -0.248 & -0.247 & -0.237 & -0.247 & -0.150 & -0.183 & -0.176 & -0.214 \\
\hline II & 0.285 & 0.272 & 0.249 & 0.260 & 0.293 & 0.289 & 0.220 & 0.239 & 0.235 & 0.255 \\
\hline III & -0.026 & -0.027 & -0.002 & -0.016 & -0.051 & -0.042 & -0.067 & -0.053 & -0.056 & -0.042 \\
\hline Substituent on carbazole & -0.611 & -0.608 & -0.608 & -0.608 & -0.603 & -0.611 & -0.078 & -0.450 & -0.446 & 0.085 \\
\hline
\end{tabular}


obvious that both Part I and Part III of each molecule are negatively charged and serve as electronic acceptors, while Part II are positively charged and serve both bridge and electronic donors. So all designed molecules could be structurally defined as the A- $\pi$-D- $\pi$-A type. In details, Part I contains much more negative charges than Part III, especially for compounds a to f which contain double nitryl substituents. The substituents on carbazole play vital roles in the charge distribution and their effects could be sorted as nitryl $>$ hydroxyl $>$ methoxy $>$ amino $>$ bromine atom.

Dipole moment $(\boldsymbol{\mu})$ : For a given molecule, the dipole moment $(\mu)$ represents its polarity and has substantial impacts on the second-order nonlinear optical property. The values of $\mu$ were generally determined by the strength of donor/acceptor, as well as the entire charge distribution. Calculated values of $\mu$ for all designed molecules were listed in Table-2. Our results show that, with the positional variation of 3,6-dinitro-N-pvinylphenyl carbazole substituent on coumarin, the values of $\mu$ for compounds a to $\mathbf{f}$ gradually increase following the order of $\mathbf{c}, \mathbf{d}, \mathbf{e}, \mathbf{b}, \mathbf{f}$, to $\mathbf{a}$. The fact should be attributed to the increasing difference in the charge distribution between Part I and Part II.

Our results also show that substituents modified on carbazole also exhibit different degrees of influences on $\mu$. For compounds $\mathbf{b}$ and $\mathbf{b} \mathbf{1}$ to $\mathbf{b 4}$, the value of $\mu$ reaches the largest when carbazole is substituted with nitryl (i.e. compounds b) and decreases following the order of b1, b4, b2, to b3. Known as a vector, $\mu$ value is equal to the charges of positive center (or negative center) multiplied by the distances between two centers (i.e. $\mu=\mathrm{q} \mathrm{L}$ ). The distance is almost identical for molecules $\mathbf{b}$ and $\mathbf{b} \mathbf{1}$ to $\mathbf{b 4}$, while the charge of Part II (positive center) of molecule $b$ is obviously larger than molecules b1 to b4. The value of $\mu$ could be modified by adjusting the ability of donor/acceptor.

Second-order nonlinear optical coefficient $(\beta)$ : In this paper, the first hyperpolarizabilities of all molecules were obtained at a static state. Results output from Gaussian03 yields 10 vector components of a $3 \times 3 \times 3$ matrix, including $\beta_{\mathrm{xxx}}$, $\beta_{x x y}, \beta_{x y y}, \beta_{y y y}, \beta_{x x z}, \beta_{x y z}, \beta_{y y z}, \beta_{x z z}, \beta_{y z z}$, and $\beta_{z z z}$. For compounds a to $\mathbf{f}$, their $\beta_{\mathrm{xxx}}$ and $\beta_{\mathrm{xyy}}$ components are much larger than the rest. On the other hand, the $\beta_{\mathrm{xxx}}$ components of compounds b1 to $\mathbf{b} 4$ are much larger than all the others. For all designed molecules, components in the $\mathrm{z}$-axis yield slight contributions to $\beta_{\text {tot }}$ due to the $\mathrm{C} 1$ symmetric molecular structure. The maximum value of $\beta$ (Table-2) are derived in the $\mathrm{x}$-axis. It reveals that the intramolecular charge transfer mainly occurs in the $\mathrm{x}$-axis and the xy-plane, which is caused by the offset of carbazole from coumarin in the xy-plane. Consequently, the secondorder nonlinear optical responses would be much more remarkable in the $\mathrm{x} / \mathrm{y}$-axis than in the $\mathrm{z}$-axis.

In Table-2, it was found that $\beta_{\text {tot }}$ value of compounds b1 to b4 (ranging from 14 to $71 \mathrm{k}$ ) are about ten times higher than those of compounds a to f. The fact, as we presumed, should be attributed to the strong electron-accepting nitryl groups modified on carbazole for compounds a to $\mathbf{f}$. The molecular structures of all designed molecules, as mentioned before, all belong to the A- $\pi$-D- $\pi$-A type. At each end of any designed molecule, Part I (carbazole together with its substituents) and Part III (coumarin) act as the electronic acceptors, respectively. Since Part III is common for all designed molecules, the intramolecular charge transfer, to a large content, was thereby mainly determined by the charge distribution of Part I and partially by its position. For compounds b1 to b4, the group of carbazole is modified with electron-donating substituents (amino, hydroxyl, methoxy, and bromine) which lower the electron-accepting capacity of Part I (Table-1). The order of corresponding $b_{\text {tot }}$ values, i.e. b1 $>$ b3 $>$ b2 $>$ b4 , is exactly consistent with that of electron-donating capacity as $-\mathrm{NH}_{2}>-\mathrm{OCH}_{3}>-\mathrm{OH}>-\mathrm{Br}$.

For a given molecule, the relation between its hyperpolarizability and its electronic transition in low-lying excited states could be briefly described by the two-level model as

$$
\beta \propto \frac{\Delta \mu_{e g} f_{e g}}{E_{g e}^{3}}
$$

where $\Delta \mu_{\mathrm{eg}}=\Delta \mu_{\mathrm{e}}-\Delta \mu_{\mathrm{g}}$ is the change of dipole moment between the ground and excited (charge transfer) state, $\mathrm{f}_{\text {eg }}$ is the oscillator strength, and $\mathrm{E}_{\mathrm{ge}}$ is the transition energy ${ }^{15,16}$. All these factors are strongly correlated and vary with electronic properties of the donor/acceptor and the length of the conjugated bridge. With the aid of Gaussian 03, the electronic spectra of designed molecules were calculated by the TD-DFT method at the B3LYP/6-31G level. Several key characteristics, e.g. $\mathrm{E}_{\mathrm{ge}}$, the maximum absorption wavelength $\left(\lambda_{\max }\right), \mathrm{f}_{\mathrm{eg}}(>0.1)$, $\Delta \mu_{\mathrm{eg}}$, and the dominant transition, are listed in Table-3. It is noteworthy that, as compared to compounds a to f, compounds b1 to b4 (especially b1) all have much larger $\Delta \mu_{\mathrm{eg}}$ values while relatively lower $\mathrm{E}_{\mathrm{ge}}$ values . Following eqn. 1, the compound of b1 was re-verified with the largest hyperpolarizability. However, it also has the largest $\lambda_{\max }(648.78 \mathrm{~nm})$ among all

\begin{tabular}{|c|c|c|c|c|c|c|c|c|c|c|c|}
\hline \multicolumn{12}{|c|}{$\begin{array}{l}\text { TABLE-2 } \\
\text { MAJOR COMPONENTS OF SECOND-ORDER NONLINEAR OPTICAL } \\
\text { COEFFICIENTS } \beta_{\text {tot }} \text { (a.u) AND DIPOLE MOMENTS } \mu \text { (Debye) OF TEN MOLECULES }\end{array}$} \\
\hline Molecule & $\beta_{x x x}$ & $\beta_{x x y}$ & $\beta_{x y y}$ & $\beta_{y y y}$ & $\beta_{x x z}$ & $\beta_{\mathrm{yyz}}$ & $\beta_{x z z}$ & $\beta_{\mathrm{yzz}}$ & $\beta_{z z z}$ & $\beta_{\text {tot }}$ & $\mu$ \\
\hline $\mathbf{a}$ & -1043.22 & -550.26 & -2896.46 & 861.36 & 201.70 & 236.80 & 42.89 & -124.48 & -29.70 & 3922.61 & 15.37 \\
\hline b & -1445.78 & 80.64 & 3147.75 & 51.02 & -213.27 & -8.58 & 11.90 & -49.64 & 114.20 & 1719.21 & 10.65 \\
\hline c & -5502.52 & -245.88 & -3085.44 & 28.77 & -374.92 & -2.40 & 254.11 & -29.24 & -59.14 & 8348.90 & 6.36 \\
\hline d & -1094.00 & 98.18 & -3016.93 & -288.81 & 169.94 & 388.61 & -55.67 & 3.27 & 36.38 & 4213.02 & 7.26 \\
\hline e & 1988.99 & 223.22 & -3192.27 & -115.01 & -60.27 & -74.25 & 4.87 & -21.21 & 13.48 & 1207.65 & 7.66 \\
\hline f & -276.30 & 343.63 & 3126.37 & -153.18 & 337.54 & -53.93 & -134.47 & 49.91 & 100.87 & 2753.22 & 13.73 \\
\hline b1 & 70121.27 & 1016.78 & 404.10 & 95.48 & 967.36 & 44.99 & -5.21 & 41.94 & 66.16 & 70538.77 & 7.50 \\
\hline b2 & 30177.33 & -330.66 & -196.76 & -58.55 & -334.73 & -40.10 & -19.44 & -46.20 & -67.69 & 29967.62 & 5.30 \\
\hline b3 & -35612.06 & -399.18 & 208.35 & -59.18 & 389.83 & 38.79 & 22.02 & -44.76 & 66.24 & 35388.93 & 5.17 \\
\hline b4 & -14453.28 & -21.89 & 361.35 & -53.64 & 12.49 & 38.19 & 8.01 & -46.27 & 71.18 & 14084.91 & 5.92 \\
\hline
\end{tabular}


COMPUTED TRANSITION ENERGY ( $\mathrm{E}_{\mathrm{ge}}$ IN eV), ABSORPTION WAVELENGTHS ( $\lambda$ IN nm), OSCILLATOR STRENGTHS $\left(f_{\mathrm{eg}}\right), \Delta \mu_{\mathrm{eg}}$, AND MAJOR CONTRIBUTIONS OF THE CRUCIAL EXCITED STATES FOR TEN MOLECULES

\begin{tabular}{|c|c|c|c|c|c|}
\hline Molecule & $\mathrm{E}_{\mathrm{ge}}(\mathrm{eV})$ & $\lambda(\mathrm{nm})$ & $f_{\text {eg }}$ & $\Delta \mu_{\mathrm{eg}}$ & Major contributions (\%) \\
\hline $\mathbf{a}$ & 3.251 & 381.35 & 0.496 & 1.132 & $\mathrm{HOMO} \rightarrow \mathrm{LUMO}+1$ \\
\hline b & 3.250 & 381.53 & 1.070 & 0.9498 & $\mathrm{HOMO} \rightarrow \mathrm{LUMO}+1(67.83)$ \\
\hline c & 3.276 & 378.45 & 0.329 & 0.7142 & $\mathrm{HOMO} \rightarrow \mathrm{LUMO}+1(68.43)$ \\
\hline d & 3.197 & 387.80 & 0.127 & 1.6196 & HOMO $\rightarrow$ LUMO (69.03) \\
\hline $\mathbf{e}$ & 3.268 & 379.39 & 0.170 & 2.1239 & HOMO $\rightarrow$ LUMO (65.49) \\
\hline f & 3.127 & 396.51 & 1.108 & 0.7923 & $\mathrm{HOMO} \rightarrow \mathrm{LUMO}+1$ (68.49) \\
\hline \multirow{2}{*}{ b1 } & 1.911 & 648.78 & 0.298 & \multirow{2}{*}{6.3556} & HOMO $\rightarrow$ LUMO (70.57) \\
\hline & 2.957 & 419.26 & 0.203 & & $\mathrm{HOMO} \rightarrow \mathrm{LUMO}+1$ (69.55) \\
\hline \multirow{2}{*}{ b2 } & 2.374 & 522.29 & 0.324 & \multirow{2}{*}{5.5759} & HOMO $\rightarrow$ LUMO $(70.36)$ \\
\hline & 3.385 & 366.31 & 0.936 & & HOMO-2 $\rightarrow$ LUMO \\
\hline \multirow{2}{*}{ b3 } & 2.294 & 540.59 & 0.323 & \multirow{2}{*}{5.7417} & HOMO $\rightarrow$ LUMO (70.39) \\
\hline & 3.323 & 373.15 & 0.485 & & $\mathrm{HOMO} \rightarrow \mathrm{LUMO}+1$ \\
\hline \multirow{2}{*}{ b4 } & 2.839 & 436.71 & 0.454 & \multirow{2}{*}{6.5332} & HOMO $\rightarrow$ LUMO (70.08) \\
\hline & 3.476 & 356.72 & 0.969 & & HOMO-1 $\rightarrow$ LUMO (69.82) \\
\hline
\end{tabular}
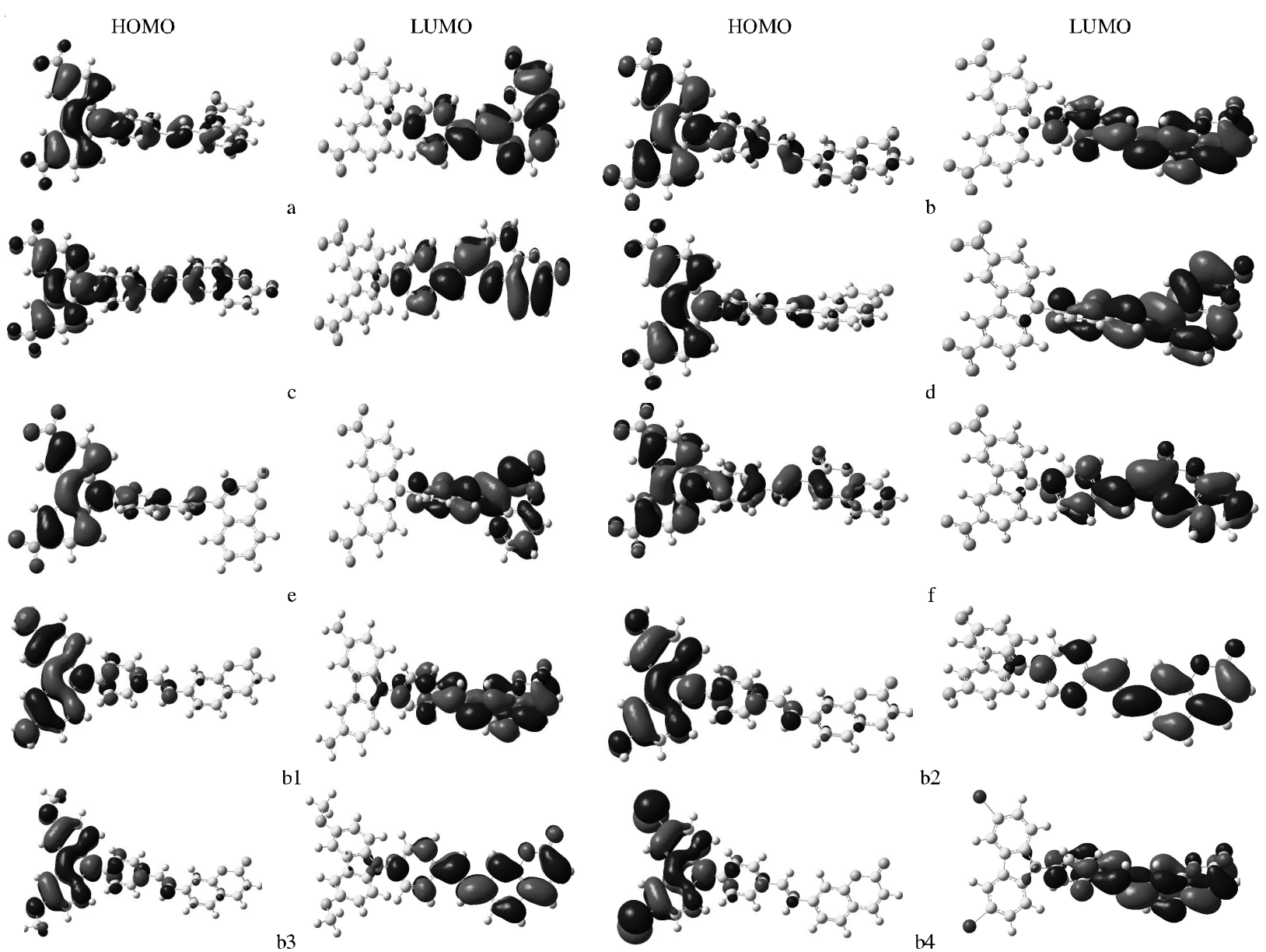

Fig. 2. Frontier molecular orbitals

designed molecules, which indicates poor light transmittance. For all the other molecules, their $\lambda_{\max }(350-400 \mathrm{~nm})$ values are restricted within the ultraviolet band and indicate excellent light transmittance.

The frontier molecular orbitals, especially HOMO and LUMO, have determinant influences on the intramolecular charge transfer and corresponding photophysical properties of compounds ${ }^{17}$. The TD-DFT calculations show that excited transitions of all molecules mainly comprise the $\mathrm{HOMO} \rightarrow$ LUMO, HOMO $\rightarrow$ LUMO +1 transitions and also some deeper layer orbitals transitions. As shown in Fig. 2, both HOMO and LUMO are $\pi$-orbitals. For all designed molecules, electrons at the LUMOs were densely located on the styryl and coumarin. On the contrary, the distribution of electrons at the HOMOs is quite different: those of compounds a to $f$ are dispersed in almost the whole molecule, while those of com- 
pounds b1 to b4 are densely concentrated around carbazole and a spot of around the styryl substituent. It is obvious that the charge transfer within compounds b1 to b4 mainly occurs from modified carbazole groups to the styryl and then to coumarin. The electron-donating substituents on carbazole of such molecules (b1 to b4) significantly enhanced the CT extent and remarkably promoted the second-order nonlinear optical properties.

\section{Conclusion}

Based on DFT method, the second-order nonlinear optical properties and relative parameters of some $\mathrm{N}$ - $p$-vinylphenyl carbazole coumarin derivatives were theoretically investigated. The following conclusions are drawn:

- All ten designed molecules could be structurally characterized as the A- $\pi$-D- $\pi$-A type.

- The substituents on carbazole, as well as the position of carbazole on coumarin, could change the conjugacy of molecules. Better conjugacy was observed for compounds b1 to b4.

- The positional variation of carbazole substituents presents remarkably affects on $\beta_{\text {tot }}$.

- Compounds b1 to b4 have larger $\beta_{\text {tot }}$ values than a to $\mathbf{f}$. The fact can be explained as an altogether result of their low transition energy, large $\Delta \mu_{\text {eg }}$ values and large CT extent.

- By introduction of electron-donating substituent (e.g. $\mathrm{NH}_{2}, \mathrm{OH}$ etc.) to carbazole, $\beta_{\text {tot }}$ value of the title coumarin is remarkably enhanced. The second-order nonlinear optical properties of them are thereby promoted.

\section{ACKNOWLEDGEMENTS}

The authors thank the National Natural Science Foundation of China (41006022) and the Taishan Scholar Program of Shandong Province for financial support of this work.

\section{REFERENCES}

1. P. Gnanasekaran and J. Madhavan, Asian J. Chem., 22, 109 (2010).

2. V.M. Geskin, C. Lambert and J.L. Bredas, J. Am. Chem. Soc., 125, 15651 (2003).

3. Z.J. Li, Z.R. Li, F.F. Wang, F. Ma, M.M. Chen and X.R. Huang, Chem. Phys. Lett., 468, 319 (2009).

4. J.L. Humphrey, K.M. Lott, M.E. Wright and D. Kuciauskas, J. Phys. Chem. B, 109, 21496 (2005).

5. R. Andreu, M.J. Blesa, L. Carrasquer, J. Garín, J. Orduna, B. Villacampa, R. Alcalá, J. Casado, M.C. Ruiz Delgado, J.T. López Navarrete and M. Allain, J. Am. Chem. Soc., 127, 8835 (2005).

6. H.T. Bai, H.C. Lin and T.Y. Luh, J. Org. Chem., 75, 4591 (2010).

7. K. Mohanalingam, P.C. Ray and P.K. Das, Synth. Met., 82, 47 (1996).

8. Q.Q. Li, C.G. Lu, J. Zhu, E.Q. Fu, C. Zhong, S.Y. Li, Y.P. Cui, J.G. Qin and Z. Li, J. Phys. Chem. B, 112, 4545 (2008).

9. F. Rong-Wei, L. Xiao-Hui, Y. Sai-Sai, J. Yu-Gang, X. Yuan-Qin and C. De-Ying, Chin. Phys. Lett., 25, 700 (2008).

10. U. Tripathy and P.B. Bisht, Chem. Phys., 125, 144502 (2006).

11. C.H. Lu, H. Zhang, S.A. Zhang and Z.R. Sun, Chin. Phys. B., 21, 123202 (2012).

12. H. Sekino, Y. Maeda and M. Kamiya, Mol. Phys., 103, 2183 (2005).

13. B. Kirtman, S. Bonness, A. Ramirez-Solis, B. Champagne, H. Matsumoto and H. Sekino, J. Chem. Phys., 128, 114108 (2008).

14. M. Kamiya, H. Sekino, T. Tsuneda and K. Hirao, J. Chem. Phys., 122, 234111 (2005).

16. J.L. Oudar and D.S. Chemla, J. Chem. Phys., 66, 2664 (1977).

17. D.R. Kanis, M.A. Ratner and T. Marks, J. Chem. Rev., 94, 195 (1994). 